\title{
Isolation of Osteosarcoma-Associated Human Antibodies from a Combinatorial Fab Phage Display Library
}

\author{
Carmela Dantas-Barbosa, ${ }^{1}$ Fabrícia P. Faria, ${ }^{2}$ Marcelo M. Brigido, $^{3}$ \\ and Andrea Q. Maranhão ${ }^{3,4}$ \\ ${ }^{1}$ Institut Gustave Roussy, UPRES EA 3535 PR2. 39, Rue Camille Desmoulins, 94805 Villejuif, France \\ ${ }^{2}$ Departamento de Bioquímica e Biologia Molecular, Instituto de Ciências Biológicas, Universidade Federal de Goiás, \\ ICB II-Campus II Samambaia, 74001-970 Goiania, GO, Brazil \\ ${ }^{3}$ Departamento de Biologia Celular, Instituto de Ciências Biológicas, Universidade de Brasília, Campus Universitário Darcy Ribeiro, \\ Asa Norte, 70910900 Brasilia, DF, Brazil \\ ${ }^{4}$ Laboratório de Imunologia Molecular, Universidade de Brasília, Campus Universitário Darcy Ribeiro, Sala ASS 128, ICC Sul, \\ Asa Norte, 70910-900 Brasilia, DF, Brazil
}

Correspondence should be addressed to Carmela Dantas-Barbosa, carmeladantas@hotmail.com

Received 8 February 2009; Revised 29 June 2009; Accepted 9 September 2009

Recommended by Anthony L. DeVico

\begin{abstract}
Osteosarcoma, a highly malignant disease, is the most common primary bone tumor and is frequently found in children and adolescents. In order to isolate antibodies against osteosarcoma antigens, a combinatorial osteosarcoma Fab library displayed on the surface of phages was used. After three rounds of selection on the surface of tumor cells, several osteosarcoma-reactive Fabs were detected. From these Fabs, five were better characterized, and despite having differences in their VH (heavy chain variable domain) and $V \kappa$ (kappa chain variable domain) regions, they all bound to a protein with the same molecular mass. Further analysis by cell ELISA and immunocytochemistry suggested that the Fabs recognize a membrane-associated tumor antigen expressed in higher amounts in neoplasic cells than in normal tissue. These results suggest that the human Fabs selected in this work are a valuable tool for the study of this neoplasia.
\end{abstract}

Copyright ( $) 2009$ Carmela Dantas-Barbosa et al. This is an open access article distributed under the Creative Commons Attribution License, which permits unrestricted use, distribution, and reproduction in any medium, provided the original work is properly cited.

\section{Introduction}

Osteosarcoma is the most common primary bone tumor that affects children and young adults [1]. It is highly malignant, characterized by a high propensity to metastasize [2]. Use of multiagent chemotherapy pre- and postoperatively has significantly improved the outcome in the last 30 years, leading to a 5-year disease-free survival for patients with localized tumors in the $20 \%$ to $60 \%$ range [3]. Despite the use of these drugs, approximately $30 \%$ of patients with localized disease and $60 \%$ of patients with pulmonary metastases succumb to the illness [4]. Osteosarcoma is a complex neoplasia. Cytogenetic analysis has revealed multiple chromosomal rearrangements without a typical translocation, as can be seen in Ewing's sarcoma. In recent years, the immunocytochemical subclassification of osteosarcomas has significantly enhanced the accuracy of pathological diagnoses [5], suggesting that new immunochemical markers could further improve diagnosis and prognosis. Recently, Salas et al. [6] introduced Ezrin expression as a prognostic factor for the survival rate of patients with conventional osteosarcoma, correlating its expression with tumor progression. It was also shown that Ezrin is useful as an immunohistochemical marker to differentiate between chondroblastic osteosarcomas and conventional chondrosarcomas [7]. However, other osteosarcoma markers are required to further characterize this complex, multifactorial neoplasia.

Antibodies to tumor mark are being used to treat different forms of cancers. Among the currently FDA approved antibodies, some of them are now under clinical trials to osteosarcoma (http://www.clinicaltrials.org/). The success of the treatment is somewhat related to the antibody origin; in 
general it is expected that humanized or fully human antibodies are better tolerated than murine monoclonal ones. To isolate such potential biopharmaceuticals, many approaches can be used, including the phage display technique. The use of the phage display technique can lead to the isolation of either antibodies or peptides able to discriminate tumor cells [8]. In a traditional approach, the identification of the tumor marker is the first step, after that it is necessary to isolate an antibody against this marker. Using a Fab phage display library, these steps can be merged into one, and it is possible to identify antigens without previous knowledge. The use of complex antigens such as cell membranes broadens the possibility of finding differentially expressed markers. However, as a more complex antigen source is used, the techniques employed to separate bound phages from unbound phages become more difficult. This usually makes selection on cell surfaces much more complex than selection using purified antigens. Focusing on this challenge, different selection procedures have been developed. Among these procedures, a method called BRASIL (Biopanning and Rapid Analysis of Selective Interacting Ligands) was specifically designed to isolate phages able to bind to the cell surface [9].

In order to isolate anti-osteosarcoma fully human Fabs, we used a previously constructed combinatorial Fab phage display library produced with antibody repertoires from osteosarcoma patients, relying on the fact that these antibody repertoires could be enriched with antibodies against the patients' own tumors [10]. Here, we report the utilization of this library for panning Fabs against the surface of osteosarcoma cells. The selection procedure was adapted from that of the BRASIL method for using a phage antibody library [9]. This is the first report of the use of the BRASIL method with a human Fab library. After three rounds of selection against the cell surface of an osteosarcoma cell line, we were able to identify many reactive Fabs, most of them sharing similar $\mathrm{V}$ genes. Five Fabs were chosen for further analysis, including western blot, cell ELISA, and immunocytochemistry. Our data revealed that these Fabs recognize a membrane antigen that is more widely expressed in the osteosarcoma cells compared to normal osteoblast and fibroblast cells. These results suggest that these human Fabs could be further used to improve the diagnosis and prognosis of osteosarcoma.

\section{Materials and Methods}

2.1. Cell Lines. The human osteosarcoma cell lines U2 OS (HTB-96) and Saos-2 (HTB-85), and the fetal normal osteoblast cell line hFOB1.19 (CRL-1172) were purchased from ATCC and cultured as recommended. A short-term culture of normal human fibroblasts was cultured at $37^{\circ} \mathrm{C}$ in 5\% CO2 with Ham's F10 medium supplemented with $100 \mathrm{U} / \mathrm{mL}$ of penicillin, $100 \mu \mathrm{g} / \mathrm{mL}$ of streptomycin, and $10 \%$ FBS.

2.2. Panning of the Fab Phage Library with Osteosarcoma Cells. A combinatorial Fab phage display library generated from the antibody $\mathrm{V}$ regions of eleven osteosarcoma patients, containing $2.7 \times 10^{8}$ different forms, was constructed previ- ously [10] and used for selection according to the BRASIL method [9]. Briefly, the cultured cells were collected with PBS and $5 \mathrm{mM}$ EDTA and then washed with the medium defined for each lineage, resuspended in culture medium containing $1 \%$ BSA at a cell density of approximately $1 \times 10^{6}$ cells per $\mathrm{mL}$, and incubated with $1 \times 10^{11}$ plaque forming units (pfu) of phage for approximately 1.5 hours on ice with sporadic gentle mixing. After that, the cell-phage suspension was transferred to the top of a nonmiscible organic lower phase (a $9: 1$ mixture of dibutyl phthalate : cyclohexane) and centrifuged at $1000 \mathrm{~g}$ for 10 minutes at room temperature. The tube was snap frozen in liquid nitrogen, and the cellphage pellet was used to infect a log-phase culture of Escherichia coli (strain XL1-Blue). In the selection procedure using the surface of U2-OS cells, we also performed a preclearing step before the third selection cycle. To do this, the phages were incubated with normal hFOB 1.19 osteoblasts, and, after separation by centrifugation through the organic phase, the supernatant containing the unbound phages (in the aqueous upper phase) was incubated with U2-OS osteosarcoma cells as described above. The selected phages were amplified by $E$. coli infection according to the procedure described elsewhere [11] and were then used in the next selection cycle.

2.3. Expression of Soluble Fab Fragments. DNA was extracted from the bacterial pellet obtained after each round of selection using a QIAprep $\mathbb{R}$ spin miniprep Kit (Qiagen), and then used to transform HB2151 E. coli cells for production of soluble Fab without the filamentous phage Gene III fusion protein partner. Individual carbenicillinresistant $\mathrm{HB} 2151$ colonies were picked and grown overnight $(\mathrm{ON})$ at $37^{\circ} \mathrm{C}$ in a 96 deep well plate in $1 \mathrm{~mL} \mathrm{SB}$ medium containing carbenicillin $(100 \mu \mathrm{g} / \mathrm{mL})$ and glucose $(1 \%)$. After this incubation, the cultures were used to produce two replica plates: one for a glycerol stock and the second for the production of the soluble Fabs themselves. The original ON culture plate was centrifuged, and plasmid DNA was extracted from the E. coli using the QIAprep $\mathbb{R}$ Kit. Fab production was performed by inoculation of $1.5 \mathrm{~mL}$ of $\mathrm{SB}$ medium containing carbenicillin $(100 \mu \mathrm{g} / \mathrm{mL})$ and glucose (1\%) with $50 \mu \mathrm{L}$ of the $\mathrm{ON}$ culture. Cultures were incubated at $37^{\circ} \mathrm{C}$, shaking at $250 \mathrm{rpm}$, until they reached an OD of 0.9 at $600 \mathrm{~nm}$. The cultures were then centrifuged, and fresh SB medium containing carbenicillin $(100 \mu \mathrm{g} / \mathrm{mL})$ and IPTG $(2 \mathrm{mM})$ was added, followed by incubation at $30^{\circ} \mathrm{C}$ for an additional 18 hours. Supernatants containing the soluble Fabs were used in the dot blot and ELISA assays.

For large-scale Fab production, the procedure was the same as described above, except that phages were produced in $100 \mathrm{~mL}$ of SB medium E. coli culture. Supernatants were concentrated using Centriprep 10 filter units (Millipore) and buffer exchanged in PBS. Purification of Fabs was performed using Ni-NTA resin (Qiagen) under nondenaturing conditions according to the manufacturer's instructions. Proteins were analyzed by SDS-PAGE [12].

2.4. Sequence Analysis. Sequencing reactions were prepared with reverse primers specific for the $\mathrm{VH}$ and VL regions 
(CH: 5'CGCCTGAGTTCCACGACACC3', MMB4: 5'GCTTCCGGCTCGTATGTTGTGT3'， C $\kappa$ : 5'AGAGGAGTCCAGATTTCA3', and MMB5: 5'CGTTTGCCATCTTTTCATAATC3') [10] and were performed using ET Terminator (GE-Healthcare) in a MegaBace 500 plus sequencer (Molecular Dynamics). Alignments were done using IgG Blast at the NCBI blast server (http://www.ncbi.nlm.nih.gov/blast) and with the program BioEdit [13]. The Kabat numbering and CDR definitions were adopted from Andrew Martin's web site (http://www.bioinf.org.uk/abs/).

2.5. Analysis of Binding of Selected Fabs to Total Protein Extracts and to the Cell Surface by ELISA. Ninety-six well plates were coated with $100 \mu \mathrm{L}$ of a $10 \mu \mathrm{g} / \mathrm{mL}$ total protein solution prepared from osteosarcoma and osteoblast cells as described in the next section. Human serum albumin was used as a mock protein. The coating was carried out overnight at $4^{\circ} \mathrm{C}$. The plates were then blocked with $3 \%$ BSA and incubated with $100 \mu \mathrm{L}$ of the induced supernatant from each selected clone. The supernatant from the empty parental plasmid (pComb3X) E. coli culture was assayed as a negative control. Fab binding was detected with a rat anti-HA (hemagglutinin decapeptide tag) HRP-conjugated antibody from Roche, followed by the ABTS peroxidase substrate. For selecting individual clones, a threshold value was arbitrarily set at ten times greater than the value obtained for the empty vector and at least three times greater than the value obtained for the incubation with proteins from normal osteoblast cells.

Cell ELISA was performed according to the protocol adapted from Williams and Sharon (2002) [14]. Briefly, polystyrene 96-well tissue culture plates were seeded with $2 \times 10^{6}$ U2-OS, Saos-2, or fibroblast cells in $200 \mu \mathrm{L}$ of culture medium defined for each cell lineage supplemented with 10\% (v/v) FBS and incubated for 30 hours. The cells were fixed with $4 \%$ formaldehyde in PBS for 20 minutes at room temperature followed by two washes with PBS. The washed cells were incubated with $100 \mu \mathrm{L}$ of the induced supernatant from each clone or with the purified Fab. The empty parental pComb3X plasmid supernatant was used as a negative control. Detection of Fab binding was performed as described above.

2.6. Immunodetection of Osteosarcoma Fab Ligands. Protein extracts from U2-OS and fibroblast cells were prepared in cold lysis buffer $(0.5 \%$ NP- $40,0.3 \%$ Triton X 100, $50 \mathrm{mM}$ Tris $\mathrm{HCl} \mathrm{pH} 8.0,5 \mathrm{mM} \mathrm{MgCl}_{2}, 0.5 \mathrm{mM}$ EDTA, $1 \mathrm{mM}$ ATP, $1 \mathrm{mM}$ DTT, $10 \%$ glycerol) and complete mini-EDTA-free protease inhibitor cocktail tablets from Roche. Total protein extracts were separated by SDS-PAGE under reducing conditions and transferred to nitrocellulose membranes (GEHealthcare). After blocking with 5\% skim milk-TBST at room temperature for 1 hour, the membranes were probed with one of the five purified Fabs $(0.02 \mu \mathrm{g} / \mathrm{mL})$ for 2 hours at RT and washed 3 times with TBST $(50 \mathrm{mM}$ Tris- $\mathrm{HCl}$, $\mathrm{pH} 7.5,150 \mathrm{mM} \mathrm{NaCl}, 0.05 \%$ tween 20 ). The membranes were then incubated with a rabbit anti-HA (hemagglutinin decapeptide tag) antibody (Santa Cruz) diluted $1: 1000$ for 1 hour, washed again and incubated with anti-rabbit HRP at a $1: 5000$ dilution. Bands were visualized using an ECL SuperSignal West Pico Chemiluminescent Substrate detection kit (Thermoscientific). To control loading differences, a rabbit anti-alpha-tubulin antibody (AbCAM) was used instead of the selected Fabs.

2.7. Immunocytochemistry. Cultures of U2-OS and human fibroblast cells grew until they reached $70-90 \%$ confluency on a slide chamber. The cells were then washed twice with PBS and fixed and permeabilized by immersion in precooled methanol for 6 minutes at $-20^{\circ} \mathrm{C}$. After two washes with PBS, the slides were incubated with $2 \%$ BSA (w/v) and 5\% normal goat serum (v/v) for 30 minutes. After an overnight incubation at $4^{\circ} \mathrm{C}$ with $15 \mu \mathrm{L}$ of purified Fabs, the slides were washed and incubated with 1:200 anti-HA antibody (Santa Cruz) for 45 minutes, followed by an incubation with a goat anti-rabbit IgG-FITC at $1: 2000$ (Sigma) in a moist chamber in the dark. After addition of DAPI I, the slides were observed with an Axiolab fluorescence microscope (Zeiss) using the Applied Imaging Cytovision software.

2.8. Immunohistochemistry. Archived, formalin-fixed, paraffin-embedded tissue sections $(4 \mu \mathrm{m})$ were deparaffinized in xylene and rehydrated using a series of alcohol and distilled water solutions. Endogenous peroxidases were quenched by incubation with $10 \mathrm{~V} \mathrm{H}_{2} \mathrm{O}_{2}$ for 10 minutes, followed by microwave antigen retrieval in $10 \mathrm{mM}$ sodium citrate buffer, $\mathrm{pH}$ 6.0. Purified anti-osteosarcoma Fabs at $200 \mu \mathrm{g} / \mathrm{mL}$, diluted to 1 : $200(\mathrm{~N}-\mathrm{H} 7)$ and 1 : $20000(\mathrm{O}-\mathrm{G} 11)$, were incubated with tissue sections for 1 hour at room temperature. After rinsing with phosphate-buffered saline, the slides were incubated for 30 minutes with rabbit antiHA antibody (Santa Cruz), diluted 1 : 200 in 1\% BSAPBS, and then incubated with a biotinylated goat antirabbit IgG (Rockland). After the antibody incubations, the slides were washed with Tris-based buffer and then incubated with streptavidin-conjugated horseradish peroxidase $\left(\mathrm{LSAB}+\mathrm{kit}^{\mathrm{TM}}\right.$, Dako) and DAB as the chromogen. The sections were counterstained with Harry's hematoxylin and covered with Entellan (Merck).

\section{Results}

3.1. Selection of Fabs that Bind to Osteosarcoma Cells. To select recombinant antibody fragments that bind to osteosarcoma cells, we used a human Fab phage display library constructed from PBMCs of eleven osteosarcoma patients [10] using two different cell lines: Saos-2 osteosarcoma cells (N Fab clones) and U2-OS osteosarcoma cells (O Fab clones). Both panning approaches were repeated three times. For the U2-OS cells, we included a negative selection cycle before the third round, in which the phages were first adsorbed to normal hFOB 1.19 osteoblast cells, followed by panning against osteosarcoma cells. One hundred eighty three clones from the second and third rounds of selection from both approaches were screened by dot immunoblot using an anti-HA antibody to identify Fab expressing clones. 
TABLE 1: Reactivity of randomly selected Fabs toward tumor antigens in ELISA assays.

\begin{tabular}{lccc}
\hline Cycle of selection(System) & Clones assayed & Positive clones in Cell ELISA $^{\mathrm{a}}$ & Positive clones on Total Protein Extracts $^{\mathrm{b}}$ \\
\hline 2nd (N) & 40 & 10 & 28 \\
3rd (N) & 39 & $\mathrm{NT}^{\mathrm{c}}$ & 13 \\
2nd (O) & 48 & 0 & 1 \\
3rd (O) & 40 & 1 & 4 \\
\hline
\end{tabular}

a A clone was considered positive if the absorbance at $405 \mathrm{~nm}$ was three times greater than the absorbance found for hFOB 1.19 cell surface binding.

${ }^{\mathrm{b}}$ A clone was considered positive if the absorbance at $405 \mathrm{~nm}$ was ten times greater than the absorbance observed for empty vector supernatant.

${ }^{\mathrm{c}} \mathrm{NT}=$ Not tested.

We found that $67 \%$ of the $\mathrm{N}$ clones and $46 \%$ of the $\mathrm{O}$ clones gave positive signals, demonstrating that these clones express reactive Fabs (data not shown). Individual Fab-expressing clones were tested for the ability to bind to osteosarcoma cell proteins by ELISA using two different strategies. The first approach evaluated Fab binding to fixed osteosarcoma cell monolayers, and the second approach evaluated Fab binding to total protein extracts from osteosarcoma cells. Seventynine clones from the $\mathrm{N}$ system and eighty-eight from the $\mathrm{O}$ system were tested. Table 1 summarizes these results. Clones showing an $\mathrm{A}_{450}$ of less than 0.1 (similar to supernatants without Fab) were considered nonbinders.

The most reactive clones were tested further in another ELISA assay using protein extract from osteosarcoma cells and normal osteoblast cells. An unrelated protein was included as a negative control. Triplicates of 27 clones were tested in this second trial, including 16 from the second and 6 from the third rounds of the $\mathrm{N}$ system and 2 clones from the second and 3 from the third round of the $\mathrm{O}$ system. The results of this assay confirmed the reactivity of the Fabs with osteosarcoma cells (Figure 1).

3.2. VH and VL Repertoire Usage of Selected Fabs. The $\mathrm{VH}$ and VL coding regions of the 20 most reactive clones were determined by sequencing (Figure 2). Analysis of the deduced amino acid sequences of the corresponding $\mathrm{V}$ genes indicated that all $\mathrm{VH}$ and $\mathrm{V} \kappa$ chains were distinct from the sequences $(51 \mathrm{VH}$ and $74 \mathrm{~V} \kappa)$ previously described from the original library of randomly chosen clones [10]. The VH sequences revealed that all clones belonged to the VH3 family and that their CDR3 varied in length from 6 to 14 amino acid residues. The majority of the clones (12/20) had a VH37 gene segment and a CDR3H of eight amino acids in length. An unusual histidine residue was predicted at position $52 \mathrm{a}$ of clone O-G11. This was first reported in the original library. The presence of the amino acid cysteine (C) at position 79 in framework 3 is a striking characteristic, and it was found in 12 clones harboring the VH3-7 gene segment, as can be seen in the N-H7 clone.

The amino acid sequences encoded by the $\mathrm{V} \kappa$ genes also differed from those reported for the unselected repertoire. The $\mathrm{O} 2 \mathrm{~V} \kappa$ family clones N-E8 and O-C10 had a conservative valine substitution at position 48 (I48V), and clones OF4 and O-G11 (B3 V $\kappa$ family) had a nonconservative phenylalanine substitution at this same position (I48F). This rare mutation is reported to occur in less than $1 \%$ of the $\mathrm{V} \kappa$ sequences [15], and was present in different clones of the original library [10]. The B3 family clones also presented a previously observed conservative change at the key position 64 (G64A). The predominance of clones harboring the VH3.7 (12/20) and A17 (11/20) gene segments supports the specific ligand selection.

Based on the results of the ELISA and the DNA sequencing, we chose five different Fabs for further analysis emphasizing their reactivity. The clones chosen were: N-H7 (VH37-A17, frequency 11/20), N-E8 (VH3-23-O2, frequency 1/20), N-H10 (VH3-20-A30, frequency 1/20), O-F4 (VH323-B3, frequency 1/20) and OG-11 (VH3-74-B3, frequency 2/20). The Fabs were produced in $100 \mathrm{~mL}$ cultures and purified using metal affinity columns before use in further analyses.

3.3. Clones N-H7, N-E8, N-H10, O-F4, and O-G11 Recognize the Same Overexpressed Osteosarcoma Protein. Immunoblot experiments were carried out for an initial characterization of the molecules in whole cell lysates derived from the osteosarcoma cell line U2-OS or from normal fibroblasts that were detected by the chosen Fabs. From this analysis, an intense $80 \mathrm{kDa}$ band and a weaker $50 \mathrm{kDa}$ band were observed for all analyzed Fabs, suggesting that all clones recognized the same proteins and that these proteins are present in both osteosarcoma cell lines (Saos-2 and U2OS). The same pattern was observed at a lower intensity when normal fibroblasts were probed, suggesting that these proteins are overexpressed in malignant bone cells (Figure 3 ).

3.4. Pattern of Fab Staining of Osteosarcoma Cells and Tissue. Further analysis of Fab reactivity was addressed by comparing the ability of the antibody fragments to bind to monolayers of osteosarcoma cells and normal fibroblasts by cell surface ELISA. Figure 4 shows the confirmation that the purified Fabs recognized a protein present in both kinds of cells (tumor and normal human fibroblast). However, the signal obtained was at least twice as strong for the tumor cells as for the normal fibroblasts, suggesting overexpression of the antigen in the osteosarcoma cells. The clones obtained from selection in Saos-2 cells (the $\mathrm{N}$ system) were tested against Saos-2 and U2-OS osteosarcoma cell lines. The clones from the $\mathrm{O}$ system were assessed using only U2-OS cells in addition to the normal human fibroblasts.

The antigen-binding specificity of each selected Fab was also evaluated by measuring the cell surface binding capacity using an immunofluorescence assay. Considering that the five Fabs apparently recognized the same protein, further 


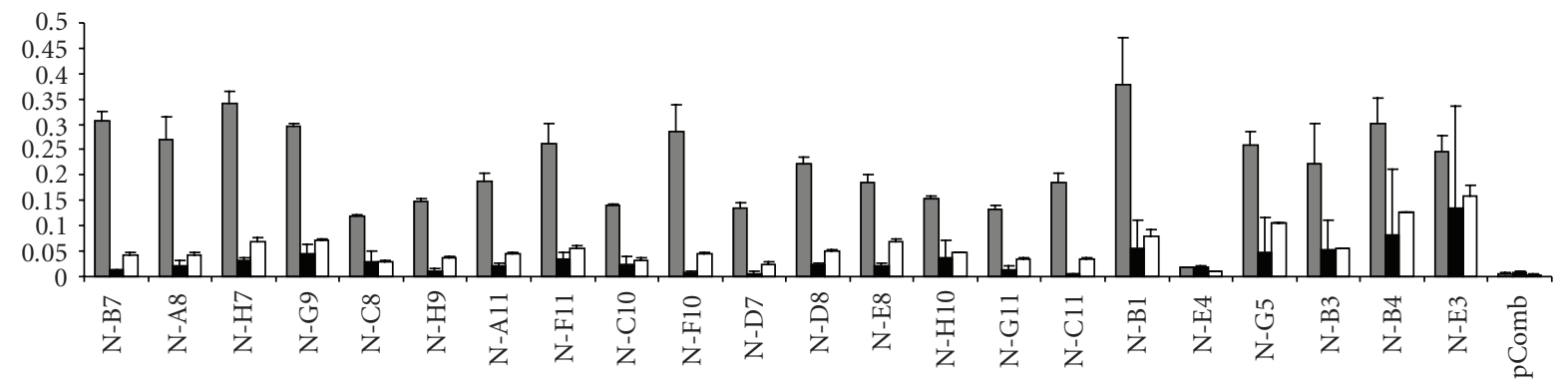

ㄴ Saos-2

- $\mathrm{hFOB}$

$\square$ HSA

(a)

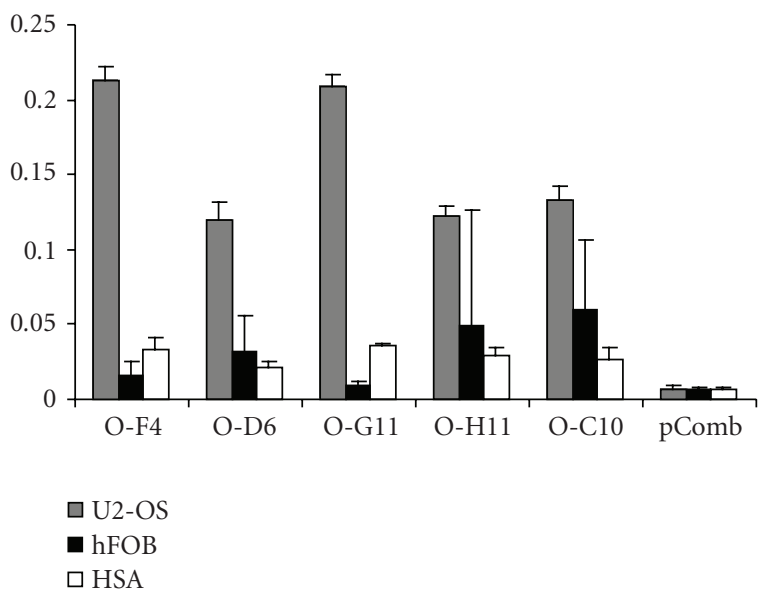

(b)

Figure 1: Selection of Fab clones able to recognize osteosarcoma cell protein extracts. Protein extracts from Saos-2 cells (horizontal line bars, panel (a)), U2-OS cells (horizontal lines, panel (b)), and h FOB 1.19 (normal human osteoblast, black bars, both panels) were used to coat a 96-well plate. E. coli supernatants from the N system (panel (a)) or the O system (panel (b)) Fab clones were added to the plate. After washing, the bound Fabs were detected using rabbit anti-HA antibody followed by an HRP-conjugated anti-rabbit antibody. The experiments were performed in triplicate. The Fabs were also added to HSA- (human serum albumin) coated wells (diagonal line bars, both panels) as a negative control. Supernatant from an empty vector E. coli culture was also used as negative control (pComb). The absorbances were measured at $405 \mathrm{~nm}$.

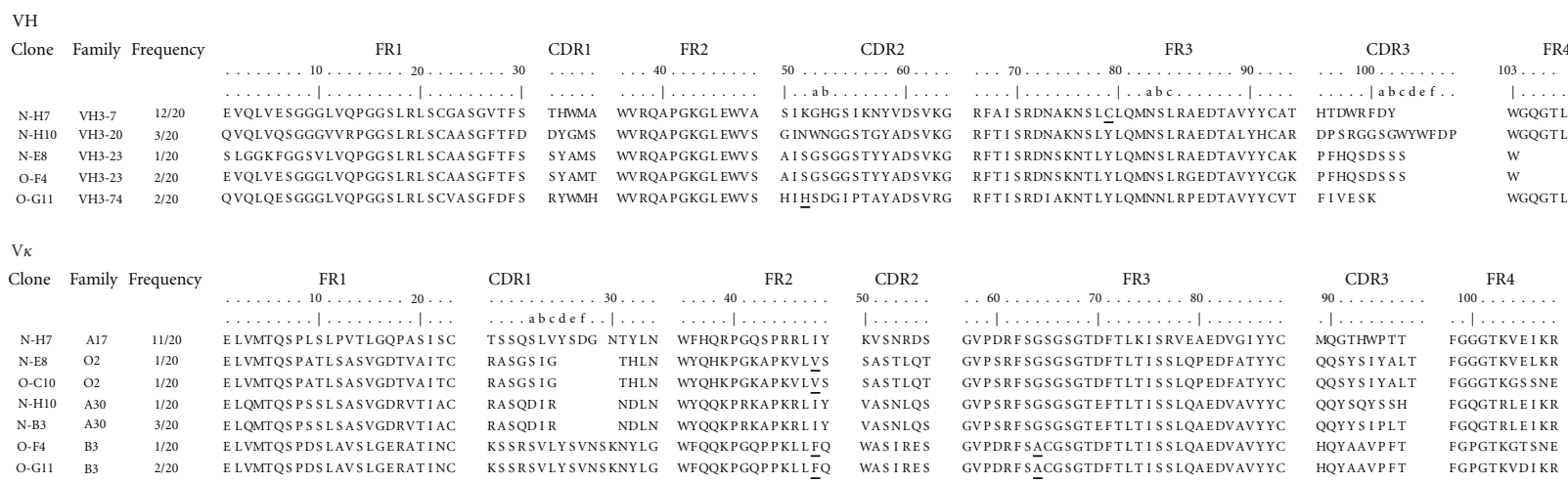

FIGURE 2: Variable gene repertoires of selected Fabs. Twenty different reactive Fab genes were sequenced, and the amino acid sequences of their VH (upper panel) and V $\kappa$ (lower panel) domains were deduced. Framework (FR) and complementary determining regions (CDR) are shown. The unusual residues are underlined. 


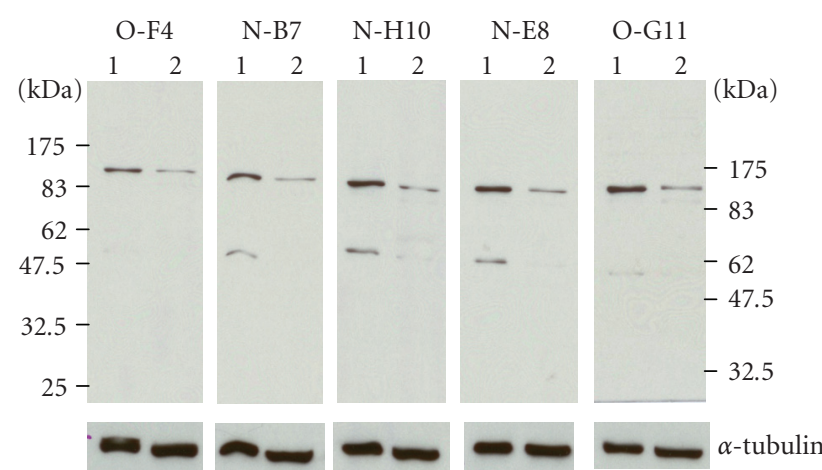

Figure 3: Detection of osteosarcoma antigens by selected Fabs. A western blot using five selected purified Fabs was performed. Protein extracts from U2-OS cells (lane 1) and normal human fibroblasts (lane 2) were transferred to nitrocellulose membranes. The membranes were probed with the O-F4, N-H7, N-H10, N-E8, and O-G11 Fabs. For loading comparison, an anti-alpha tubulin antibody was also used.

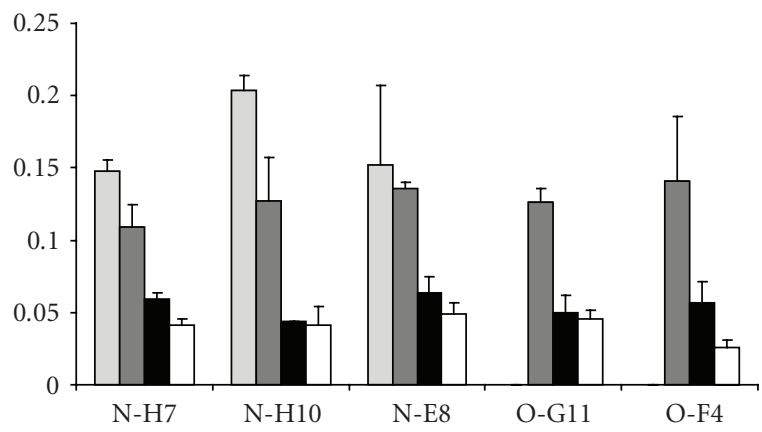

FIgUre 4: Cell surface ELISA. The selected purified Fabs were added to monolayers of Saos-2 cells (light gray bars), U2-OS cells (dark gray bars), and human fibroblasts (black bars). The bound Fabs were detected using rabbit anti-HA and HRP-conjugated antirabbit antibodies. Human serum albumin (white bars) was used as negative control. The absorbances were measured at $405 \mathrm{~nm}$.

investigations were done mainly with the $\mathrm{N}-\mathrm{H} 7$ (Figure 5, panels (a) and (b)) and O-G11 (Figure 5, panel (c), (d), (e) and (f)) Fabs. These Fabs were chosen based on the frequency of $\mathrm{N}-\mathrm{H} 7$, whose VH and VL sequences were shared with 10 other selected clones, and on singularity of O-G11, as it came from the third round of the $\mathrm{O}$ system, after depletion on normal osteoblast cells. The immunofluorescence staining pattern suggested that these two Fabs interact with a cell membrane component. The staining was dot like for the osteosarcoma cell line U2-OS (Figure 5, panels (b) and (d)), but only a vague staining was observed for the normal fibroblasts (Figure 5, panels (a) and (c)).

Immunohistochemistry analyses performed using archived normal bone and osteosarcoma sections confirmed the membrane staining pattern. Panels (e) and (f) of Figure 5 show the reactivity of the O-G11 Fab in a normal bone section and in an osteosarcoma section, respectively. There was visible staining in the bone marrow adipocytes and in the osteoblasts in the normal tissue (Figure 5,

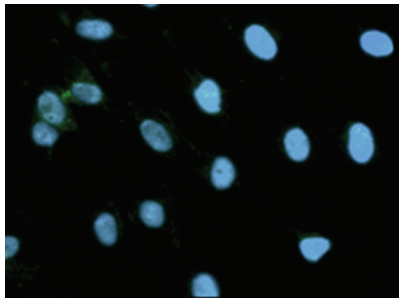

(a)

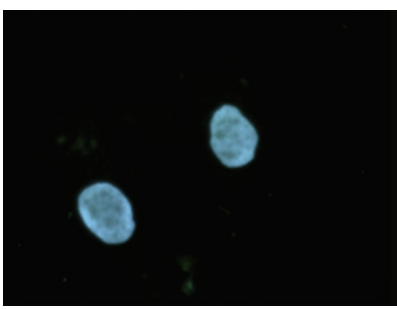

(c)

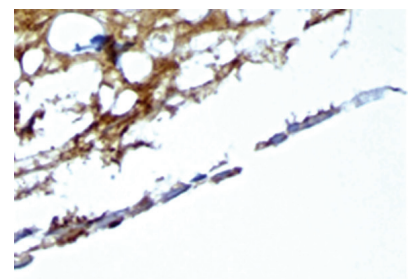

(e)

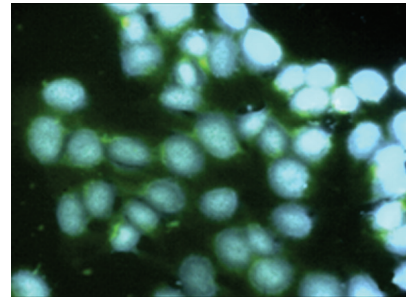

(b)

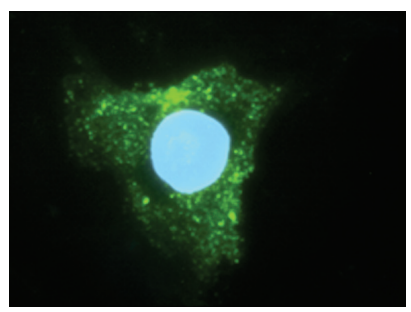

(d)

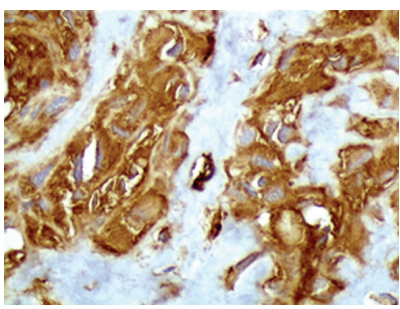

(f)
FIGURE 5: Indirect immunofluorescence staining and immunohistochemistry were performed with the selected Fabs. Methanol-fixed fibroblasts ((a) and (c)) or U2-OS osteosarcoma cells ((b) and (d)) were stained with Fab N-H7 ((a) and (b)) or Fab O-G11 ((c) and (d)). Fab O-G11 was also used to stain to normal bone (e) (200x) and osteosarcoma tissue (f) (400x) sections. The osteoblasts in normal bone are arranged in lines; the empty region corresponds to bone trabecules dislocated during washing. These results are representative from four different osteosarcoma cases analyzed.

panel (e)), but staining was less intense than the staining observed in osteosarcoma tissues (Figure 5, panel (f)). This demonstrated the preference of O-G11 for tumor cell membranes.

\section{Discussion}

This work has focused on the isolation of antibodies against osteosarcoma cells using a human Fab phage display library that was constructed from antibody repertoires of osteosarcoma patients. The selection was accomplished using whole cells as the antigen source, with the goal of isolating novel antiosteosarcoma antibodies. This strategy poses an advantage over the traditional strategy using protein extracts, as the library probes the cell surface allowing the identification of protein and nonprotein antigens [16]. Despite some intrinsic difficulties, such as the binding to a rare antigen and the loss of nonbinder phages associated with this approach [17], successful examples have been reported. 
Among the successful approaches using whole cells is the BRASIL method, described as a very efficient way to rescue ligand phages [9]. Until now, this technique has been used only with peptide libraries [18, 19]. We successfully adapted the BRASIL protocol for use with a Fab library. We performed three rounds of selection, and the reactivity analysis of the second and third rounds revealed that after two rounds we were able to identify Fabs that bind to osteosarcoma cells. The ability to select specific tumor binders is usually attributed to the depletion step [20], but the omission of this step does not impair the isolation of specific binders [21, 22]. Our data suggest that the introduction of a negative selection step (only before the third selection cycle for the $\mathrm{O}$ system) did not significantly improve the isolation of specific Fabs, since the $\mathrm{N}$ and $\mathrm{O}$ Fabs apparently recognized the same antigen (Figure 3 ). The lack of more effective selection in the $\mathrm{O}$ system could also be a consequence of the small number of positive clones in round 2 , just prior to the depletion step (Table 1).

Selected Fab clones were chosen based on their Fab coding sequences (Figure 2) and on their reactivity to tumor antigens assayed as a monolayer of osteosarcoma cells (Table 1) and total protein extracted from cells (Table 1, and Figure 1). These assays were carried out to favor the binding of antibodies that recognize the physiological conformations of the antigen(s). The five clones that were further characterized all recognized the same antigen (Figure 3), despite the fact that some of them reacted differently in the cell ELISA or protein ELISA assays, suggesting that these Fabs may recognize different epitopes [23]. Comparing the ELISA results (Figures 1 and 4), we observed that the results were similar if we used fibroblasts or normal osteoblasts, as negative controls.

Another important finding was that the same clones were recovered after selection. Also, it is remarkable that the majority of these clones (11 out of 20) harbored the same sequence (A17, for $\mathrm{V} \kappa$ and $\mathrm{VH} 3.7$ for the heavy chain, Figure 2). This cannot be attributed to a bias in the Fab library, since all of the sequenced clones from rounds two or three had different $\mathrm{VH}$ and $\mathrm{V} \kappa$ sequences compared to the original library [10]. The emergence of clones with a restricted diversity of $\mathrm{V}$ gene fragments is a feature that accounts for the selection of specific ligands, as was reported when other antibody phage display libraries were used to isolate high affinity immunoglobulins $[22,24]$. Clones sharing VH3-7 and A17 were the majority of the clones identified in our selection process. There was one exception among the 20 sequenced clones: clone N-B1, which has the VH3-7 gene associated with an A30 V $\kappa$ gene. The effect of this rare association on affinity and selectivity can be further addressed.

Apparently, all of the five chosen clones, each representing a distinct gene family, recognized the same protein profile. This was also true for Fabs selected against different osteosarcoma cell lines, Saos2 and U2-OS (clones from the $\mathrm{N}$ and $\mathrm{O}$ systems, resp.). Similar results have been reported by Aryee and coworkers regarding six different scFvs selected against FLI1 [25]. Our data point to the existence of an immunodominant antigen. The immunodominance phe- nomenon has been frequently observed in phage display analysis. In two separate studies with melanoma, one using a scFv phage display library constructed from blood from melanoma patients and the other using an Fab library, the authors were able to isolate only one positive $\mathrm{scFv}$ and Fab, respectively $[26,27]$. This is in agreement with our results using two different cell lines, Saos-2 and U2-OS, as the antigen source. This does not exclude the possibility of binding to different epitopes on the same protein, and further epitope mapping must be carried out. Another interesting characteristic was the ability of the selected antibody to bind to the antigen in both native and denaturing conditions (Figures 1(a), 1(b) and 3), suggesting a linear rather than a conformational epitope.

The immunofluorescence staining pattern (Figure 5) was typical for Fabs recognizing a membrane-associated ligand, as expected for a cell surface selection procedure. The background in immunohistochemestry was high, and although it was possible to see staining of normal cells, the staining was stronger with the osteosarcoma tissues. The OG11 Fab gave a very strong background even when used as much as 1 : 20000 dilution (estimated concentration of $0.1 \mathrm{ng} / \mathrm{mL}$ ). Usually the concentration of commercial antibodies is about $200 \mu \mathrm{g} / \mathrm{mL}$, and they are used at dilutions of $1: 50-1: 200$ in immunohistochemistry assays. O-G11 recognized the osteoblasts in a very strong way and also reacted with adipocytes in the bone medulla but did not stain the blood vessel (data not shown).

It is difficult to measure the strength of the signal in osteosarcoma cells and compare it to the signal strength from normal controls in cell imaging approaches, such as immunofluorescence and immunohistochemistry. This became more evident when a quantitative assay was employed (Figure 3). The higher level of the antigen in the osteosarcoma cells was confirmed by cell surface ELISA (Figure 4). In these assays our results showed that the antigen recognized by these Fabs was expressed at a level two times higher in the osteosarcoma cells than in normal tissue. This antigen is a potential marker for osteosarcoma, and investigation into the identity of this antigen should be completed.

In conclusion, this work describes the isolation of five different human Fabs that recognize osteosarcoma-associated antigens. These monoclonal antibody fragments harbored different VH and VL domains but apparently recognized the same protein. Our data suggest that this protein epitope is found in lower amounts in normal cell lines than in osteosarcoma cell lines and is an immunodominant antigen. As these are fully human antibodies, they can be further characterized, aiming their use as biopharmaceuticals.

\section{Acknowledgments}

The authors are grateful to Olivier Coux and Veronique Baldin for contribution on Fab purification and for U2-OS protein extracts. They are also indebted to Dr. Jorge Kalil Gibram for helpful discussion about immunohistochemistry. The comments and help from Ricardo Giordano were also valuable in the selection procedure. This work was supported by $\mathrm{CNPq}$. 


\section{References}

[1] H. D. Dorfman and B. Czerniak, "Bone cancers," Cancer, vol. 75, no. 1, pp. 203-210, 1995.

[2] D. C. Dahlin and K. K. Unni, "Osteosarcoma of bone and its important recognizable varieties," The American Journal of Surgical Pathology, vol. 1, no. 1, pp. 61-72, 1977.

[3] G. Saeter, "Osteosarcoma: ESMO clinical recommendations for diagnosis, treatment and follow-up," Annals of Oncology, vol. 18, supplement 2, pp. ii77-ii78, 2007.

[4] P. A. Meyers and R. Gorlick, "Osteosarcoma," Pediatric Clinics of North America, vol. 44, no. 4, pp. 973-989, 1997.

[5] Z. Pohar-Marinšek, "Difficulties in diagnosing small round cell tumours of childhood from fine needle aspiration cytology samples," Cytopathology, vol. 19, no. 2, pp. 67-79, 2008.

[6] S. Salas, C. Bartoli, J.-L. Deville, et al., "Ezrin and alphasmooth muscle actin are immunohistochemical prognostic markers in conventional osteosarcomas," Virchows Archiv, vol. 451, no. 6, pp. 999-1007, 2007.

[7] S. Salas, G. De Pinieux, A. Gomez-Brouchet, et al., "Ezrin immunohistochemical expression in cartilaginous tumours: a useful tool for differential diagnosis between chondroblastic osteosarcoma and chondrosarcoma," Virchows Archiv, vol. 454, no. 1, pp. 81-87, 2009.

[8] R. Brissette, J. K. A. Prendergast, and N. I. Goldstein, "Identification of cancer targets and therapeutics using phage display," Current Opinion in Drug Discovery and Development, vol. 9, no. 3, pp. 363-369, 2006.

[9] R. J. Giordano, M. Cardó-Vila, J. Lahdenranta, R. Pasqualini, and W. Arap, "Biopanning and rapid analysis of selective interactive ligands," Nature Medicine, vol. 7, no. 11, pp. 1249$1253,2001$.

[10] C. Dantas-Barbosa, M. M. Brígido, and A. Q. Maranhão, "Construction of a human Fab phage display library from antibody repertoires of osteosarcoma patients," Genetics and Molecular Research, vol. 4, no. 2, pp. 126-140, 2005.

[11] C. Rader, R. P. Steinberger, and C. F. Barbas III, "Selection from antibody libraries," in Phage Display : A Laboratory Manual, C. F. Barbas III, D. R. Burton, J. K. Scott, and G. J. Silverman, Eds., pp. 10.2-10.20, Cold Spring Harbor Laboratory Press, Cold Spring Harbor, NY, USA, 1st edition, 2000.

[12] J. Sambrook and D. W. Russel, Molecular Cloning: A Laboratory Manual, Cold Spring Harbor Laboratory Press, New York, NY, USA, 3rd edition, 2001.

[13] T. A. Hall, "BioEdit: a user-friendly biological sequence alignment editor and analysis," Nucleic Acids Symposium Series, vol. 41, pp. 95-98, 1999.

[14] B. R. Williams and J. Sharon, "Polyclonal anti-colorectal cancer Fab phage display library selected in one round using density gradient centrifugation to separate antigen-bound and free phage," Immunology Letters, vol. 81, no. 2, pp. 141-148, 2002.

[15] I. M. Tomlinson, J. P. L. Cox, E. Gherardi, A. M. Lesk, and C. Chothia, "The structural repertoire of the human $\mathrm{V}_{(\kappa)}$ domain," The EMBO Journal, vol. 14, no. 18, pp. 4628-4638, 1995.

[16] S. Mao, C. Gao, C.-H. L. Lo, P. Wirsching, C.-H. Wong, and K. D. Janda, "Phage-display library selection of highaffinity human single-chain antibodies to tumor-associated carbohydrate antigens sialyl Lewisx and Lewisx," Proceedings of the National Academy of Sciences of the United States of America, vol. 96, no. 12, pp. 6953-6958, 1999.
[17] B. M. Edwards, S. H. Main, K. L. Cantone, S. D. Smith, A. Warford, and T. J. Vaughan, "Isolation and tissue profiles of a large panel of phage antibodies binding to the human adipocyte cell surface," Journal of Immunological Methods, vol. 245, no. 1-2, pp. 67-78, 2000.

[18] P. U. Ardelt, C. G. Wood, L. Chen, et al., "Targeting urotheliumml: ex vivo assay standardization and selection of internalizing ligands," Journal of Urology, vol. 169, no. 4, pp. 1535-1540, 2003.

[19] W.-D. Jia, H.-C. Sun, J.-B. Zhang, et al., "A novel peptide that selectively binds highly metastatic hepatocellular carcinoma cell surface is related to invasion and metastasis," Cancer Letters, vol. 247, no. 2, pp. 234-242, 2007.

[20] U. B. Rasmussen, V. Schreiber, H. Schultz, F. Mischler, and K. Schughart, "Tumor cell-targeting by phage-displayed peptides," Cancer Gene Therapy, vol. 9, no. 7, pp. 606-612, 2002.

[21] T. Oyama, K. F. Sykes, K. N. Samli, J. D. Minna, S. A. Johnston, and K. C. Brown, "Isolation of lung tumor specific peptides from a random peptide library: generation of diagnostic and cell-targeting reagents," Cancer Letters, vol. 202, no. 2, pp. 219230, 2003.

[22] R. Mutuberria, S. Satijn, A. Huijbers, et al., "Isolation of human antibodies to tumor-associated endothelial cell markers by in vitro human endothelial cell selection with phage display libraries," Journal of Immunological Methods, vol. 287, no. 1-2, pp. 31-47, 2004.

[23] H. Wu, J. D. Pancook, G. Beuerlein, et al., "Cloning, isolation and characterization of human tumor in situ monoclonal antibodies," Cancer Immunology, Immunotherapy, vol. 51, no. 2, pp. 79-90, 2002.

[24] Y. Shen, X. Yang, N. Dong, X. Xie, X. Bai, and Y. Shi, "Generation and selection of immunized Fab phage display library against human B cell lymphoma," Cell Research, vol. 17, no. 7, pp. 650-660, 2007.

[25] D. N. T. Aryee, M. Kreppel, R. Bachmaier, et al., "Single-chain antibodies to the EWS $\mathrm{NH}_{2}$ terminus structurally discriminate between intact and chimeric EWS in Ewing's sarcoma and interfere with the transcriptional activity of EWS in vivo," Cancer Research, vol. 66, no. 20, pp. 9862-9869, 2006.

[26] X. Cai and A. Garen, "Anti-melanoma antibodies from melanoma patients immunized with genetically modified autologous tumor cells: selection of specific antibodies from single-chain Fv fusion phage libraries," Proceedings of the National Academy of Sciences of the United States of America, vol. 92, no. 14, pp. 6537-6541, 1995.

[27] S. Pereira, H. Maruyama, D. Siegel, et al., "A model system for detection and isolation of a tumor cell surface antigen using antibody phage display," Journal of Immunological Methods, vol. 203, no. 1, pp. 11-24, 1997. 

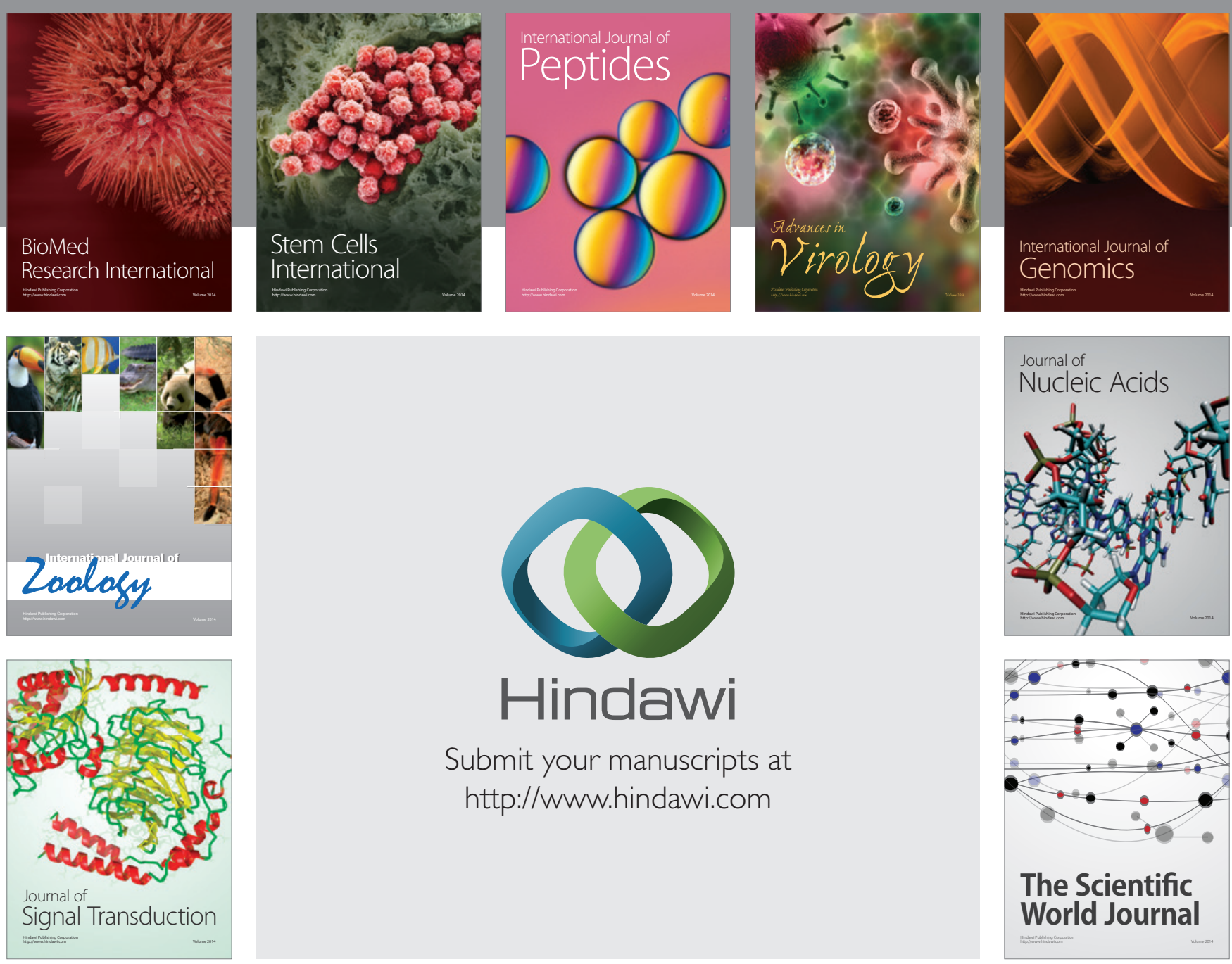

Submit your manuscripts at

http://www.hindawi.com
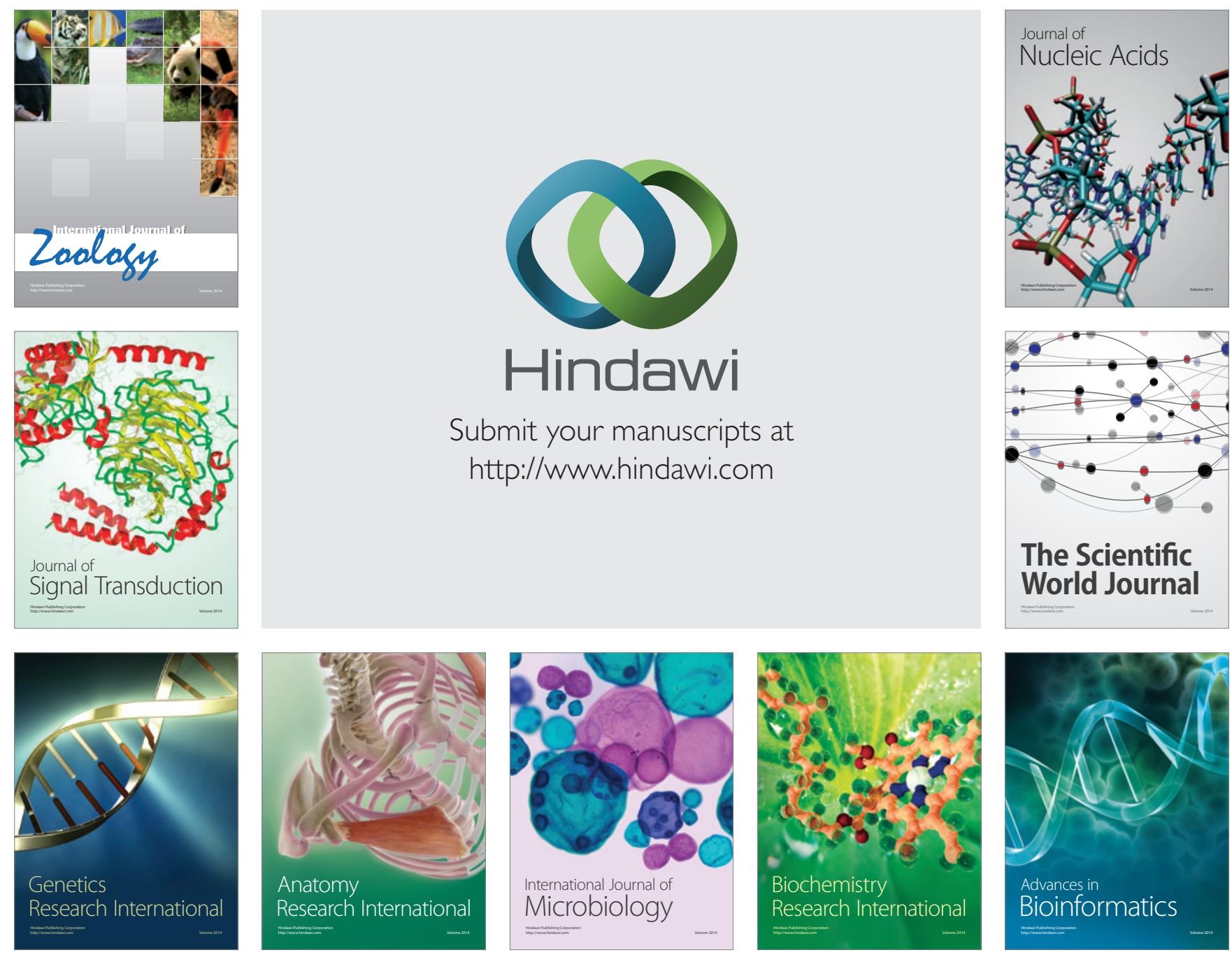

The Scientific World Journal
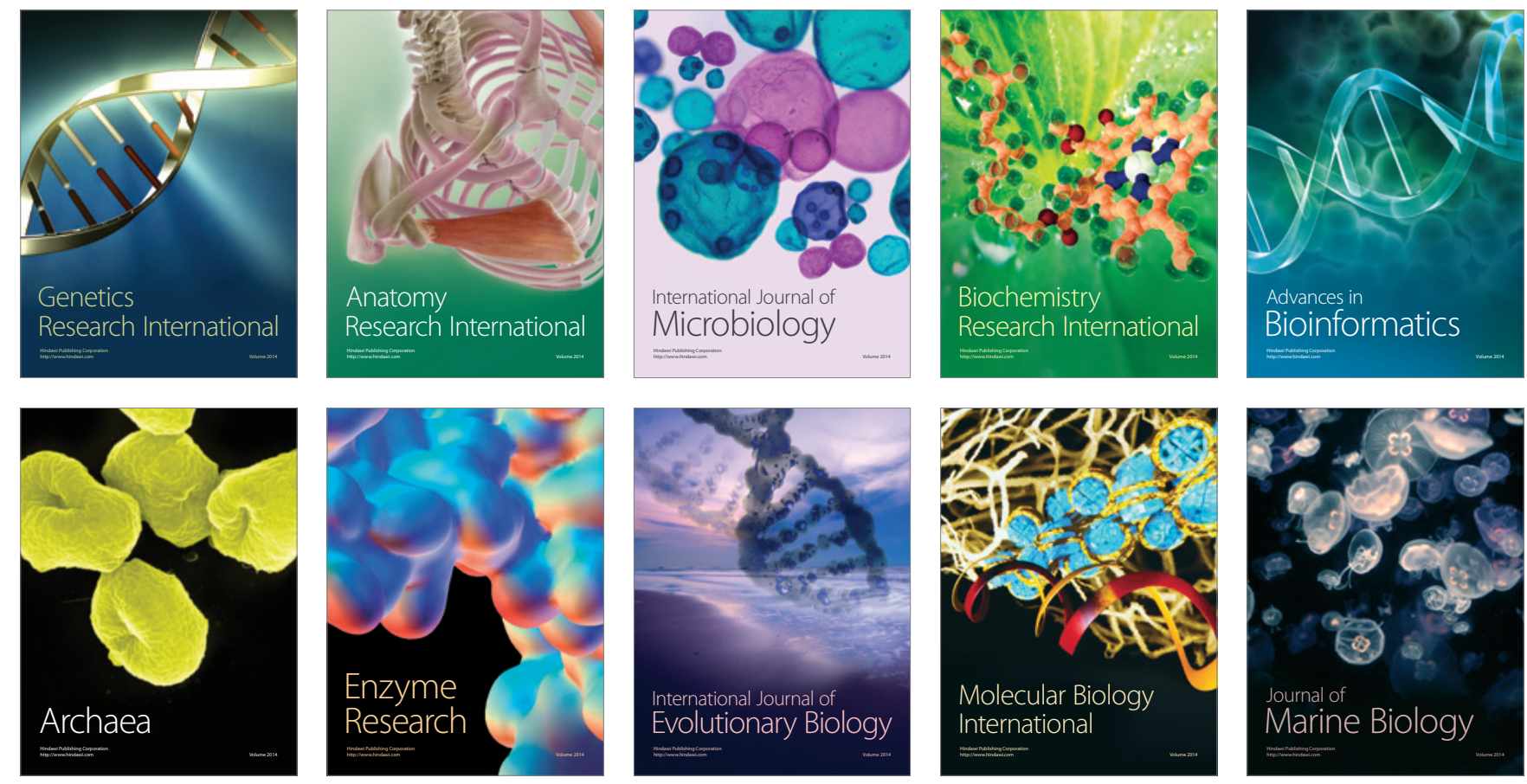